\title{
HADRON PRODUCTION IN VIRTUAL PHOTON-PHOTON ANNIHILATION
}

\author{
G. KÖPP \\ III. Physikalisches Institut der TH Aachen \\ T.F. WALSH and P. ZERWAS ${ }^{+}$ \\ Deutsches Elektronen-Synchrotron DESY, Hamburg
}

Received 25 October 1973

\begin{abstract}
In this article, we systematically study the amplitudes for annihilation of two virtual photons into hadron resonances and continuum states, a process measurable with colliding electron-positron beams. The asymptotic behavior of resnance form factors and their absolute scale follows from light cone or parton model considerations; definite predictions for inclusive two-photon annihilation are derived by the same methods.
\end{abstract}

\section{Introduction}

The discovery of scaling properties in inelastic electron and neutrino scattering [1] has stimulated interest in the interaction of electromagnetic and weak currents with hadrons. Under certain kinematical conditions these reactions with high mass currents probe the light cone structure of current products [2]. Far-reaching consequences can be derived by requiring the currents to build up a closed algebraic sys. tem on the light cone [3]. A physical realization of these ideas is the so-called parton model [4], the central feature of which is the existance of fundamental constituents of both the hadronic matter and the electromagnetic and weak currents. The prescriptions of the parton model are not precise, and the model has therefore a certain heuristic value.

If the main point of deep inelastic scattering processes is the constituent structure of the electromagnetic and weak currents, then it is natural to study in as much detail as possible those processes which involve only currents or current products. This is the major motivation for studying such processes as $\mathrm{e}^{+}+\mathrm{e}^{-} \rightarrow \gamma^{*} \rightarrow$ hadrons, where $\gamma^{*}$ denotes a photon with large virtual mass. It is important to give a theoretical description of such current reactions, based on the above mentioned ideas, in the expectation that a confrontation with experiment will increase our understanding of deep inelastic processes.

\footnotetext{
${ }^{+}$Permanent Address: III. Phys. Institut, TH Aachen
} 
There are two classes of processes in which one measures matrix elements of current products without the interference of hadrons in the initial state: single-photon $\mathrm{e}^{+} \mathrm{e}^{-}$annihilation into an unanalyzed hadron system, as mentioned above, which measures the vacuum expectation value of the product of two electromagnetic currents [5], and two-photon annihilation into an unanalyzed hadron system, which measures the vacuum expectation value of the product of four currents [6]. Unfortunately, similar processes involving weak currents seem to be inaccessible*. Besides these fully inclusive reactions, one can study semi-inclusive processes like $\mathrm{e}^{+} \mathrm{e}^{-} \rightarrow \mathrm{h}+\mathrm{X}$, where $\mathrm{h}$ is an observed hadron and $\mathrm{X}$ is summed over. These processes are complicated for a simple reason. Whereas the crossed process $\mathrm{e}^{-}+\overline{\mathrm{h}} \rightarrow \mathrm{e}^{-}+\mathrm{X}$ reveals the constituent structure of $\overline{\mathrm{h}}$, the semi-inclusive annihilation process probes the complicated final state interaction of the basic current constituent. This reflects itself in the fact that, in the parton model, the Bjorken variable in the semi-inclusive process is actually the Feynman scaling variable $p_{\mathrm{h}} / p_{\mathrm{h} \text {, max }}$ in the process parton + antiparton $\rightarrow h+X$. For this reason we believe that the reaction studied here - the annihilation of two massive photons in to hadrons - provides a cleaner test of current theoretical ideas than semi-inclusive $\mathrm{e}^{+} \mathrm{e}^{-}$annihilation, and offers good possibilities for gaining an understanding of deep inelastic processes.

Our aim here will be to provide a theoretical picture of the two-photon process as well as some specific predictions based on the parton model or the quark light-cone algebra. Some of the results may hold more generally and not just within this context; others not. For this reason we give, after a general review of kinematics, a qualitative discussion of models. Then we turn to resonance production, $\gamma^{*}+\gamma^{*} \rightarrow$ resonance or, equivalently, the absorptive part of $\gamma^{*} \gamma^{*} \rightarrow \gamma^{*} \gamma^{*}$ forward scattering in the resonance region. Finally we discuss the high energy limit of the absorptive part of off-shell photon-photon scattering. A few of these results can already be found in the literature [6]. However, the purpose of this article is to present a coherent picture of the processes under discussion, so we shall repeat material where necessary.

The process in which one can measure $\gamma^{*}\left(q_{1}\right)+\gamma^{*}\left(q_{2}\right) \rightarrow \mathrm{X}(P)$ is

$$
\mathrm{e}^{-}\left(p_{1}\right)+\mathrm{e}^{ \pm}\left(p_{2}\right) \rightarrow \mathrm{e}^{-}\left(p_{1}^{\prime}\right)+\mathrm{e}^{ \pm}\left(p_{2}^{\prime}\right)+\mathrm{X}(P),
$$

where the energy and angle of the scattered electrons determine the independent variables of the basic process, $q_{1}^{2}, q_{2}^{2}, P^{2}$. This reaction has attracted interest in connection with nearly real photon-photon scattering $\left(q_{1}^{2} \sim q_{2}^{2} \sim-m_{\mathrm{e}}^{2}\right)$ [7]. In this kinematic region, the cross section is enhanced by a factor $\sim\left(\ln E / m_{\mathrm{e}}\right)^{2}$ relative to the case in which the photon masses are finite. We shall not discuss here the measurability question in any detail. The reason is that in the kinematic region of interest to us this process looks (in the parton model) like the pure QED process $\mathrm{e}^{-} \mathrm{e}^{ \pm} \rightarrow \mathrm{e}^{--} \mathrm{e}^{ \pm} \mu^{+} \mu^{-}$, which will be discussed elsewhere. Of course, the experiments required to study reactions are not trivial, but we do not believe that they are insupport-

* The reader can amuse himself by considering the Primakoff reaction $\nu+Z \rightarrow \mu+$ hadrons $+Z$, which involves both the electromagnetic and weak currents. 


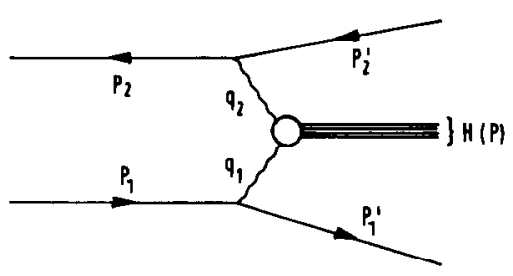

Fig. 1. Hadron production by annihilation of two virtual photons which are produced in colliding electron-positron beams.

ably difficult, either. The main problem is that the cross sections are small; this probably sets a minimum of $\sim 10^{32} \mathrm{~cm}^{-2} \cdot \mathrm{sec}^{-1}$ on the machine luminosity required to perform an experiment. However, even crude total cross sections in the appropriate kinematic region would be of great value, and we shall see that a dramatic dependence of the cross sections on a certain scaling variable emerges from parton model or light cone considerations. This can also be used as a test of these ideas, without requiring a great deal of data. Although the radiative corrections to the process $\mathrm{e}^{-} \mathrm{e}^{ \pm} \rightarrow \mathrm{e}^{-} \mathrm{e}^{ \pm} \mathrm{X}$ present a formidable problem, they should not obscure the essential features.

\section{Kinematics}

The virtual photon pair which annihilates into hadrons of even charge conjugation can be generated in a pair of colliding electron and positron beams (fig. 1). From the diagram we read off the production amplitude

$$
T \propto e^{4} \bar{u}\left(p_{1}^{\prime}\right) \gamma_{\mu} u\left(p_{1}\right) \bar{v}\left(p_{2}\right) \gamma_{\nu} v\left(p_{2}^{\prime}\right) \frac{1}{q_{1}^{2}} \frac{1}{q_{2}^{2}} T_{\mathrm{H}}^{\mu \nu}\left(q_{1}, q_{2}\right),
$$

where the amplitude $T_{\mathrm{H}}^{\mu \nu}$ is defined as*

$$
T_{\mathrm{H}}^{\mu \nu}\left(q_{1}, q_{2}\right)=i \int \mathrm{d}^{4} x \mathrm{e}^{-\frac{1}{2} i\left(q_{1}-q_{2}\right) x}\left\langle H(P)\left|T^{*}\left\{J^{\mu}\left(\frac{1}{2} x\right), J^{\nu}\left(-\frac{1}{2} x\right)\right\}\right| 0\right\rangle .
$$

In the c.m. system of the virtual photons, which is identical to the rest frame of the hadron system, we may define the helicity amplitudes

$$
T_{\mathrm{H}}^{\lambda_{1} \lambda_{2}}\left(q_{1}, q_{2}\right)=T_{\mathrm{H}}^{\mu \nu}\left(q_{1}, q_{2}\right) \epsilon_{\mu}^{\lambda_{1}}\left(q_{1}\right) \epsilon_{\nu}^{\lambda_{2}}\left(q_{2}\right), \quad \lambda_{1,2}= \pm, 0 .
$$

Four-momenta and polarization vectors are defined to be (fig. 2)

\footnotetext{
${ }^{*}$ Single-particle states are normalized by $\left\langle p, \lambda \mid p^{\prime}, \lambda^{\prime}\right\rangle=2 E \delta_{3}\left(p-p^{\prime}\right) \delta \lambda \lambda^{\prime}$.
} 


$$
\begin{aligned}
& q_{1}=\left(q_{1}^{0}, 0,0, q_{1}^{3}>0\right), \quad\left\{\begin{array}{l}
\epsilon^{ \pm}\left(q_{1}\right)=\frac{1}{\sqrt{2}}(0, \mp 1,-i, 0) \\
\epsilon^{0}\left(q_{1}\right)=\frac{1}{\sqrt{-q_{1}^{2}}}\left(q_{1}^{3}, 0,0, q_{1}^{0}\right)
\end{array}\right. \\
& q_{2}=\left(q_{2}^{0}, 0,0,-q_{1}^{3}\right), \quad\left\{\begin{array}{l}
\epsilon^{ \pm}\left(q_{2}\right)=\frac{1}{\sqrt{2}}(0, \pm 1,-i, 0) \\
\epsilon^{0}\left(q_{2}\right)=\frac{1}{\sqrt{-q_{2}^{2}}}\left(q_{1}^{3}, 0,0,-q_{2}^{0}\right)
\end{array}\right.
\end{aligned}
$$

The number of independent helicity amplitudes can be reduced by invoking the known invariance principles. To that end, we assume that the state $H$ has a definite spin and parity. Then we have from:

(i) Bose statistics :

$$
T_{\mathrm{H}}^{\lambda_{1} \lambda_{2}}\left(q_{1}, q_{2}\right)=T_{\mathrm{H}}^{\lambda_{2} \lambda_{1}}\left(q_{2}, q_{1}\right)
$$

(ii) rotational invariance :

$T_{\mathrm{H}}^{\lambda_{1} \lambda_{2}} \neq 0$ only if $\left|\lambda_{1}-\lambda_{2}\right| \leqslant J_{\mathrm{H}}$,

(iii) parity : $T_{\mathrm{H}}^{\lambda_{1} \lambda_{2}}=n_{\mathrm{H}} T_{\mathrm{H}}^{-\lambda_{1}-\lambda_{2}}, n_{\mathrm{H}}=$ normality of $H$,

(iv) time reversal :

$$
T_{\mathrm{H}}^{\lambda_{1} \lambda_{2}}=\left\{T_{\mathrm{H}_{T}}^{\lambda_{1} \lambda_{2}}\right\}^{*}
$$

Therefore, the number of independent amplitudes depends on the spin and normality of the resonance; we have

$$
\begin{array}{ll}
T_{\mathrm{H}}^{++}= \pm T_{\mathrm{H}}^{--}, T_{\mathrm{H}}^{00}= \pm T_{\mathrm{H}}^{00}: & J_{\mathrm{H}} \geqslant 0, \\
T_{\mathrm{H}}^{+0}= \pm T_{\mathrm{H}}^{-0}, T_{\mathrm{H}}^{0+}= \pm T_{\mathrm{H}}^{0-}: & J_{\mathrm{H}} \geqslant 1, \\
T_{\mathrm{H}}^{+-}= \pm T_{\mathrm{H}}^{-+}: & J_{\mathrm{H}} \geqslant 2,
\end{array}
$$

where the upper (lower) sign refers to even (odd) normality. As trivial examples, we mention the pion with one form factor, the $J^{P}=0^{+} \epsilon$-meson with two, and the $J^{P}=2^{+}$f-meson with five independent form factors. In our case the photons are both spacelike and the form factors are real.

Summing over all hadronic final states, the following tensors enters the total cross section:

$$
\vec{e}_{2} \uparrow\left\{\begin{array}{l}
q_{2}=\left(q_{2}^{0}, 0,0,-q_{1}{ }^{3}\right) \\
p=(M, \overrightarrow{0}) \\
q_{1}=\left(q_{1}^{0}, 0,0, q_{1}{ }^{3}\right)
\end{array}\right.
$$

Fig. 2. Kinematics in the hadron rest frame 


$$
\begin{aligned}
W^{\mu^{\prime} \nu^{\prime} \mu \nu}= & (2 \pi)^{4} \sum_{H} \delta_{4}\left(q_{1}+q_{2}-P_{\mathrm{H}}\right) T_{\mathrm{H}}^{\mu^{\prime} \nu^{\prime *}} T_{\mathrm{H}}^{\mu \nu} \\
= & \int \mathrm{d}^{4} x \mathrm{~d}^{4} y \mathrm{~d}^{4} z \mathrm{e}^{-i\left\{\frac{1}{2}\left(q_{2}-q_{1}\right) x-\frac{1}{2}\left(q_{2}-q_{1}\right) y+\left(q_{2}+q_{1}\right) z\right\}} \\
& \times\left\langle 0\left|\bar{T}^{*}\left\{J^{\mu^{\prime}}\left(\frac{1}{2} x\right) J^{\nu^{\prime}}\left(-\frac{1}{2} x\right)\right\} T^{*}\left\{J^{\mu}\left(\frac{1}{2} y+z\right) J^{\nu}\left(-\frac{1}{2} y+z\right)\right\}\right| 0\right\rangle,
\end{aligned}
$$

and the helicity representation,

$$
W^{m^{\prime} n^{\prime} m n}=\epsilon_{\mu^{\prime}}^{* m^{\prime}} \epsilon_{\nu^{\prime}}^{* n^{\prime}} W^{\mu^{\prime} \nu^{\prime} \mu \nu} \epsilon_{\mu}^{m} \epsilon_{\nu}^{n} .
$$

This tensor can be interpreted as the absorptive part of the off-shell photon-photon forward scattering amplitude. This is restricted by the invariance principles to satisfy:

(i) Bose statistics:

$$
W^{m^{\prime} n^{\prime} m n}\left(q_{1} q_{2}, q_{1} q_{2}\right)=W^{n^{\prime} m^{\prime} m n}\left(q_{2} q_{1}, q_{1} q_{2}\right) \text { etc. }
$$

(ii) rotational invariance : $W^{m^{\prime} n^{\prime} m n}=0$ unless $m^{\prime}-m=n^{\prime}-n$.

(iii) parity : $W^{m^{\prime} n^{\prime} m n}=(-)^{m^{\prime}-n^{\prime}}(-)^{m-n} W^{-m^{\prime}-n^{\prime}-m-n}$.

(iv) time reversal : $W^{m^{\prime} n^{\prime} m n}=W^{m m n^{\prime} n^{\prime}}$.

(v) hermeticity of $J^{\mu}$ : $W^{m^{\prime} n^{\prime} m n}=\left\{W^{m n m^{\prime} n^{\prime}}\right\}^{*}$.

Thus, there are eight helicity amplitudes (all real), which can be chosen to be [8]:

$$
\begin{array}{ll}
w^{++++}, & w^{++--}, w^{+-+-}, w^{+0+0}, \\
w^{0+0+}, & w^{0+-0}, w^{++00}, w^{0000} .
\end{array}
$$

If this absorptive part in the low-energy region is given by the contribution of one resonance of normality $n_{\mathrm{r}}$ then we get one further relation,

$$
W_{\mathrm{r}}^{++++}=n_{\mathrm{r}} W_{\mathrm{r}}^{++--} \text {. }
$$

The amplitudes are all dimensionless, as is easily checked.

The independent variables in these processes are the masses of the photons, $q_{1}^{2}<0, q_{2}^{2}<0$ and the c.m. energy of the $\gamma^{*} \gamma^{*}$ system, $s=P^{2}=\left(q_{1}+q_{2}\right)^{2}>0$. From these quantities we can construct two independent dimensionless variables [9]. The limit $q_{2}^{2}=0$ suggests defining a "Bjorken" variable

$$
\omega=1-s /\left(q_{1}^{2}+q_{2}^{2}\right),
$$

which is a constant in any limit where $\left|q_{1}^{2}+q_{2}^{2}\right| \rightarrow \infty$ with a fixed ratio to $s$. As a second variable we define

$$
\xi=\left(q_{2}^{2}-q_{1}^{2}\right) /\left(q_{2}^{2}+q_{1}^{2}\right) .
$$

In a slightly different fashion we can define the scaling variable 


$$
\xi^{\prime}=\nu / Q^{2}
$$

where

$$
\left.\begin{array}{l}
P=q_{2}+q_{1} \\
Q=\frac{1}{2}\left(q_{2}-q_{1}\right)
\end{array}\right\} \nu=P Q .
$$

This is related to the former ones by

$$
\xi^{\prime}=\xi /\left[\frac{1}{2}(\omega+1)\right] \text {, }
$$

and $\xi$ and $\xi^{\prime}$ become identical for $\left|q_{1}^{2}+q_{2}^{2}\right| \rightarrow \infty$ with $s$ fixed.

According to which variable is held fixed, several a priori inequivalent limits may be approached in off-shell photon-photon scattering. The limit $q_{1}^{2} \rightarrow \infty$ at fixed $s$ and $\xi$ we shall call the $L$ kimit, and the double limit $\lim _{s \rightarrow \infty} \lim _{q_{i} \rightarrow-\infty}$ at fixcd $\xi$ wc shall call the DL limit [10]. The limit $q_{i}^{2} \rightarrow-\infty$ at fixed $\omega$ and $\xi$ will be called the $S$ limit. The two former limits arise from light cone considerations and the DL limit is formally the threshold $\omega \rightarrow 1$ of the $S$. limit. There is a class of limits where the quantity $s / q_{1}^{2} q_{2}^{2} \rightarrow \infty$ with both $s$ and $q_{i}^{2}$ approaching infinity; we shall call this the scaling Regge limit, SR. The proper Regge limit R corresponds to $s \rightarrow \infty$ with $q_{i}^{2}$ fixed. Again formally, the SR limit corresponds to $\omega \rightarrow+\infty$ in the S limit. The reason for this abundance of limits is that we expect different behaviour in each of them as we shall see in the next section. A schematic representation of the limits is given in fig. 3 .

\section{The parton model}

The problem is now to determine the amplitudes from some physical starting point. In order to gain an overview of this problem, we discuss the basic features of the quark parton model in the covariant formulation of Landshoff, Polkinghorne and Short [4] (LPS).

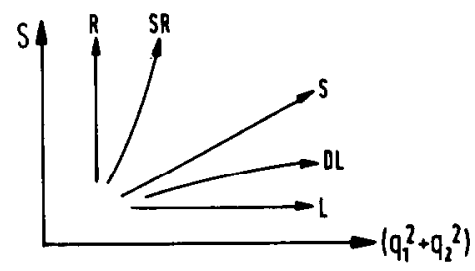

Fig. 3. Representation of the different limits in the $\left(s,\left|q_{1}^{2}+q_{2}^{2}\right|\right)$ plane. 


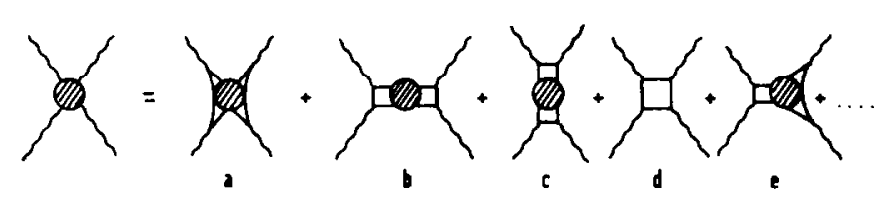

Fig. 4. Graphical representation of the connected and disconnected parts of the photon-photon scattering amplitude in the quark-parton model.

The diagrams for $\gamma^{*} \gamma^{*} \rightarrow \gamma^{*} \gamma^{*}$ in the parton model are shown in fig. 4 . The electromagnetic current is supposed to couple to fundamental fermion constituents (quarks), and the decomposition on the right-hand side in this figure is into diagrams without and with connnected quark-quark scattering amplitudes. These amplitudes include the attached quark propagator and incorporate the fundamental assumption of the model in the LPS version: all such amplitudes decrease rapidly as the squared four-momentum carried by any quark line connected to the hadron blob becomes large. As a consequence, amplitudes in which a photon couples to a fully connected blob (figs. $4 a, e)$ Decrease rapidly as the $q^{2}$ of that photon becomes large. The dominant diagrams are then $4 \mathrm{~b}, 4 \mathrm{c}$ and $4 \mathrm{~d}$ plus those obtained by crossing (we omit the latter for clarity). In several of the limits of interest to us, one or more of the three remaining amplitudes may either vanish or be non-dominant.

A possibly unpleasant feature of the model is that when taking discontinuities one appears to have free quarks in the final hadron state. Even if there were no pole in the quark propagator, it is not clear that one does not have an isolated set of states (jet) of quark quantum numbers. Because of this feature of the usual parton models we shall refer to them collectively as "disconnected".

Models in which one assumes that the quarks are massive particles can trivially escape this sort of objection [12]; one then has only diagram (c) contributing to the discontinuity, with the entire contribution coming from a connected diagramcontrary to the spirit of the parton model. The amplitude for $\mathrm{e}^{+} \mathrm{e}^{-}$annihilation provides a clear example of this. In any quark model, the diagrams are as in fig. 5 . As $q^{2} \rightarrow \infty$ the parton model has 5(a) vanishing, with the entire contribution coming from diagram 5(b). This gives the familiar result $\sigma\left(\mathrm{e}^{+} \mathrm{e}^{-} \rightarrow\right.$ hadrons $)=$

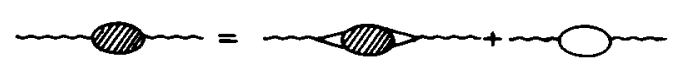

Fig. 5. Graphical representation of the connected and disconnected pieces of the photon propagator in the quark-parton model. 
$=\Sigma_{i} \mathrm{e}_{i}^{2} \sigma\left(\mathrm{e}^{+} \mathrm{e}^{-} \rightarrow \mu^{+} \mu^{-}\right)$where $e_{i}$ are the quark charges. In a massive quark model due to Bohm, Joos and Krammer [12] diagram 5(b) vanishes when $q^{2}<4 M_{q}^{2}$ where $M_{\mathrm{q}}$ is the physical quark mass; the entire contribution from the connected amplitude consists of a series of $J^{P}=1^{-}$resonances giving $\sigma\left(\mathrm{e}^{+} \mathrm{e}^{-} \rightarrow\right.$ hadrons $)=R \sigma\left(\mathrm{e}^{+} \mathrm{e}^{-} \rightarrow\right.$ $\rightarrow \mu^{+} \mu^{-}$) with $R \neq 0$. The relation $R=\Sigma e_{i}^{2}$ does not appear to arise naturally in this model, and a relation to the light-cone algebra is not evident. Although we shall have nothing further to say about such models, we wish to emphasize that many of the following results may emerge in such an approach, though these "connected" quark models have not yet been studied in detail*.

Henceforth we shall confine ourselves to the "disconnected" quark parton models. They provide a realization of the quark light cone algebra within the context of which most of the following results are model independent.

We have now to consider the behavior of the three amplitudes $5 \mathrm{~b}, \mathrm{c}, \mathrm{d}$ in the various limits of interest to us. In all those limits for which $\left|q_{1}^{2}\right|$ and $\left|q_{2}^{2}\right|$ increase as fast or faster than $s$ (L, DL and S) diagram $4 \mathrm{~b}$ vanishes essentially because of a $t_{\text {min }}$ effect: the momentum transfer along a quark propagator into the connected quarkquark scattering amplitude increases without bound according to

$$
\left|P_{\mathrm{q}}^{2}\right| \geqslant \frac{1}{2}\left|s-q_{1}^{2}-q_{2}^{2}-\sqrt{\left(s-q_{1}^{2}-q_{2}^{2}\right)^{2}}-4 q_{1}^{2} q_{2}^{2}\right| .
$$

Because the amplitudes decrease rapidly under such conditions, we conclude that diagram $4 \mathrm{~b}$ does not contribute to the limits just emtioned. In the L limit, both diagrams $4 \mathrm{c}$ and $4 \mathrm{~d}$ can contribute. The latter involves resonances in the quark-antiquark amplitude; the former can contribute to a non-resonant background. In the DL and $S$ limits diagram $4 c$ vanishes and finite contributions come solely from the disconnected diagram 4d. In the DL limit this is just the light cone algebra contribution [6]. In the scaling Regge limit, where $s / q_{1}^{2} q_{2}^{2}$ increases at the same time that $s$ and $\left|q_{1}^{2}\right|$ and $\left|q_{2}^{2}\right|$ increase, diagram $4 \mathrm{~b}$ does survive and is expected to dominate; the contribution of $4 \mathrm{~d}$ amounts to a measly fixed cut at $J=0$ in the $J$-plane expansion.

The theoretical prescription for evaluating the diagrams is the light cone expansion for the time ordered product of two currents [2], [3]:

$$
\begin{aligned}
T^{*}\left\{J_{\mu}(x) J_{\nu}(y)\right\}= & -\left[\left(\partial / \partial x_{\rho}\right) D_{\mathrm{F}}(x-y)\right] \\
& \times s_{\mu \rho \nu \sigma}\left[J_{Q^{2}}^{\sigma}(x \mid y)-J_{Q^{2}}^{\sigma}(y \mid x)\right]- \\
& -i \epsilon_{\mu \rho \nu \sigma}\left[J_{Q^{2}}^{5 \sigma}(x \mid y)+J_{Q^{2}}^{5 \sigma}(y \mid x)\right], \\
& s_{\mu \rho \nu \sigma}=g_{\mu \rho} g_{\nu \sigma}+g_{\nu \rho} g_{\mu \sigma}-g_{\mu \nu} g_{\rho \sigma},
\end{aligned}
$$

\footnotetext{
* A phenomenological scheme for such models has been proposed by Preparata [12].
} 
where $J_{Q^{2}}^{\sigma}(x \mid y)$ and $J_{Q^{2}}^{5 \sigma}(x \mid y)$ are bilocal vector and axial currents given in the free quark model by

$$
J_{Q^{2}}^{(5) \sigma}(x \mid y)=: \bar{q}(x) \gamma^{\sigma}\left(\gamma_{5}\right) q(y):
$$

The quark charge matrix is $Q$ and $Q^{2}=\frac{1}{3} Q+\frac{2}{9}$ is a combination of octet and singlet pieces. The usual currents are the local limits of the bilocal operators. This algebraic structure survives in the gluon model, where a phase factor given in terms of a line integral over the gluon field appears in the definition of the bilocal operators [3]. We assume that the bilocal is the sum of a $c$-number piece singular on the light cone plus an operator piece which has finite matrix elements.

The fully disconnected diagram fig. $4 \mathrm{~d}$ corresponds to a $c$-number piece in the commutator of two bilocals; it has the most singular behavior on the light cone and dominates in the DL limit. In the parton model this is just the discontinuity of the box graph in massless quantum electrodynamics.

\section{Resonance form factors}

The most interesting question to be answered when $s$ is held fixed concerns the asymptotic behavior of the resonance form factors in the light cone limit $q_{1}^{2}, q_{2}^{2} \rightarrow$ $\rightarrow-\infty$. In the quark model only quark spin-singlet states with negative parity and quark spin-triplet states with positive parity are able to couple to two photons; the states at low $s$ are

$$
\begin{array}{ll}
{ }^{1} \mathrm{~S}_{0} \text { with } J^{\mathrm{pc}}=0^{-+}: & \pi^{0}, \eta, \mathrm{X}^{0}(965), \\
{ }^{3} \mathrm{P}_{0} \text { with } J^{\mathrm{pc}}=0^{++}: & \pi_{\mathrm{N}}^{0}(975), \epsilon(700), \mathrm{S}^{*}(1070), \\
{ }^{3} \mathrm{P}_{0} \text { with } J^{\mathrm{pc}}=1^{++}: & \mathrm{A}_{1}^{0}(1070)(?), \\
{ }^{3} \mathrm{P}_{2} \text { with } J^{\mathrm{pc}}=2^{++}: & \mathrm{A}_{2}^{0}(1310), \mathrm{f}(1270), \mathrm{f}^{\prime}(1515) .
\end{array}
$$

This classification scheme covers all the experimentally observed low-mass mesons with positive charge conjugation.

In order to avoid considering this large number of states, we will discuss some selected examples in detail. It will then be clear how to deal with the remainder, on the basis of the quark model (including mixing effects).

\section{1. $\pi^{0}$ meson}

The $\pi^{0} \gamma^{*} \gamma^{*}$ form factor is defined as

$$
T_{\pi}^{\mu \nu}=\epsilon^{\mu \nu \rho \sigma} Q_{\rho} P_{\sigma} F_{\pi}\left(q_{1}^{2}, q_{2}^{2}\right)
$$

and is related to the only non-vanishing helicity amplitude $T_{\pi}^{++}=-T_{\pi}^{-}$by 


$$
T_{\pi}^{++}=i \sqrt{\nu^{2}-m_{\pi}^{2} Q^{2}} F_{\pi}\left(q_{1}^{2}, q_{2}^{2}\right) .
$$

In the L limit, $q_{1}^{2} \rightarrow-\infty, q_{2}^{2} \rightarrow-\infty$ with $q_{1}^{2} / q_{2}^{2}$ kept fixed we then get a simple representation of the form factor in terms of the bilocal operator matrix elements,

$$
\epsilon^{\mu \nu \rho \sigma} Q_{\rho} P_{\sigma} F_{\pi} \rightarrow \epsilon^{\mu \nu \rho \sigma} \int \mathrm{d}^{4} x \mathrm{e}^{i Q x}\left[\partial_{\rho} D_{\mathrm{F}}(x)\right]\left\langle\pi^{0}(P)\left|J_{5 \sigma}^{Q^{2}}\left(\frac{1}{2} x \mid-\frac{1}{2} x\right)+(x \leftrightarrow-x)\right| 0\right\rangle .
$$

The invariant decomposition of the bilocal matrix element for $x^{2}=0$ reads

$$
\left\langle\pi^{0}(P)\left|J_{5 \sigma}^{Q^{2}}\left(\frac{1}{2} x \mid-\frac{1}{2} x\right)+J_{5 \sigma}^{Q^{2}}\left(-\frac{1}{2} x \mid \frac{1}{2} x\right)\right| 0\right\rangle=i P_{\sigma} f_{1}(x P)+x_{\sigma} f_{2}(x P),
$$

where only $f_{1}$ contributes to the asymptotic behavior of $F_{\pi}$. Introducing the Fourier transform

$$
f_{1}(x P)=\int_{-1}^{+1} \mathrm{~d} u \mathrm{e}^{\frac{1}{2} i(P x) u} \varphi_{\pi}(u),
$$

where the (even) spectral function $\varphi_{\pi}(u)$ vanishes for $|u|>1$ from the Jost-Lehmann Dyson representation, one finds for the asymptotic expression

$$
F_{\pi}\left(q_{1}^{2}, q_{2}^{2}\right) \rightarrow \frac{1}{Q^{2}} \hat{f}_{\pi}\left(\xi^{\prime}\right)
$$

where

$$
\hat{f}_{\pi}\left(\xi^{\prime}\right)=\int_{-1}^{+1} \mathrm{~d} u \frac{\varphi_{\pi}(u)}{1+\xi^{\prime} u}=\int_{-1}^{+1} \mathrm{~d} u \frac{\varphi_{\pi}(u)}{1-\xi^{\prime 2} u^{2}} .
$$

A sum rule for $\varphi_{\pi}(u)$ follows from the fact that the bilocal current operator $J_{Q^{2}}^{5 \sigma}\left(\frac{1}{2} x \mid-\frac{1}{2} x\right)$ approaches the local operator $J_{Q^{2}}^{5 \sigma}(x)$ for $x \rightarrow 0$, the isovector part of which is related to the axial vector current in the weak interactions. We can thus express $f_{1}(0)$ in terms of the $\pi_{\ell 2}$ decay constant $f_{\pi}$ defined by $\left\langle\pi^{0}(P)\left|J_{5 \sigma}\right| 0\right\rangle=i P_{\sigma} f_{\pi}$. Then,

$$
\int_{-1}^{+1} \mathrm{~d} u \varphi_{\pi}(u)=\hat{f}_{\pi}(0)=\frac{2}{3} f_{\pi} .
$$

These results for $\hat{f}_{\pi}\left(\xi^{\prime}\right)$ are due to Brandt and Preparata, as is the so-called "smooth threshold assumption" which ensures that $\hat{f}_{\pi}( \pm 1)$ is finite [13], [14]. Since $\varphi_{\pi}(u)$ is the absorptive part of the amplitude for $\mathrm{e}^{-}+\pi^{0} \rightarrow \mathrm{e}^{-}+\mu^{+} \mu^{-}$in the scaling region with $u=1$ the threshold value, these authors suggested that the rapid decrease of the SLAC scaling functions at threshold indicated that discontinuities like $\varphi_{\pi}(u)$ also should vanish rapidly at threshold. Accepting this, we anticipate that $\hat{f}_{\pi}\left(\xi^{\prime}\right) \sim \hat{f}_{\pi}(0)$ for some range of $\xi^{\prime}$. On this basis, we have given cross section estimates in an earlier paper for the reaction $\mathrm{e}^{+}+\mathrm{e}^{-} \rightarrow \mathrm{e}^{+}+\mathrm{e}^{-}+\pi^{0}$ [6].

Note that it would not be meaningful to apply dimensional analysis here, since a dimensional scale factor $f_{\pi}$ enters explicitly. This arises from the fact that the 


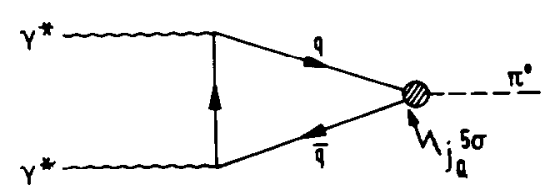

Fig. 6. Quark-parton diagram for the asymptotic $\pi^{0} \gamma^{*} \gamma *$ vertex.

photon-quark interaction in the light cone algebra is that in massless quantum electrodynamics but that the scale of the $q \bar{q} \pi^{0}$ vertex is set by the dimensional constant $f_{\pi}$; this dimensional scale then carries over into the $\pi^{0} \gamma^{*} \gamma{ }^{*}$ vertex (fig. 6).

An estimate of the structure functions $\hat{f}_{\eta}\left(\xi^{\prime}\right)$ and $\hat{f}_{\mathrm{X}}\left(\xi^{\prime}\right)$ may be found from the quark model. Let us ignore mixing effects, which can be easily incorporated. We then identify $\eta$ and $\mathrm{X}^{0}$ with pure octet and singlet states; the $\mathrm{M} \gamma^{*} \gamma^{*}$ form factors are proportional to $\operatorname{tr}\left\{\lambda^{\mathrm{M}} Q^{2}\right\}$ where $\lambda^{\mathrm{M}}$ is the $\lambda$ - matrix attached to the meson $\mathrm{M}$,

$$
\lambda^{\pi^{0}}=\frac{1}{\sqrt{2}}\left\|\begin{array}{ccc}
1 & & \\
& -1 & 0
\end{array}\right\|, \quad \lambda^{\eta}=\frac{1}{\sqrt{6}}\left\|\begin{array}{ccc}
1 & & \\
& 1 & \\
& & 0
\end{array}\right\|, \quad \lambda^{\mathrm{X}=\frac{1}{\sqrt{3}}}\|\| \begin{array}{ccc}
1 & & \\
& 1 & \\
& & 1
\end{array} \| \text {. }
$$

We immediately get the result

$$
\hat{f_{\pi}}\left(\xi^{\prime}\right): \hat{f}_{\eta}\left(\xi^{\prime}\right): \hat{f}_{\mathrm{X}}\left(\xi^{\prime}\right)=\frac{1}{\sqrt{2}}: \frac{1}{\sqrt{6}}: \frac{2}{\sqrt{3}} .
$$

These relations should be fulfilled at least as well as those for the on-shell $\gamma \gamma$ couplings when mixing is properly taken into account.

We close this subsection by noting that in the scaling limit

$$
\begin{aligned}
T^{++} & =-i \xi^{\prime} \hat{f}\left(\xi^{\prime}\right), \\
W^{++ \pm \pm} & = \pm \xi^{\prime 2}\left\{\hat{f}\left(\xi^{\prime}\right)\right\}^{2} \delta\left(s-m_{\mathrm{M}}^{2}\right) .
\end{aligned}
$$

The $\xi^{\prime 2}$ dependence of $W$ is a dramatic feature of the quark light-cone algebra. By the smooth threshold assumption, one should be able to test these relations by integrating over a range of $\xi^{\prime}$; it is not necessary to extract $f(0)$. Of course, it would be interesting to check the $\xi^{\prime}$ dependence.

\section{2. $\epsilon-m e s o n$}

We follow the prescription just given also for this case of scalar meson production. We now have two independent from factors, since parity invariance no longer requires $T_{\epsilon}^{00}$ to vanish. The form factors can be defined as

$$
T_{\epsilon}^{\mu \nu}=i A^{\mu \nu} F_{\epsilon}\left(q_{1}^{2}, q_{2}^{2}\right)+i A^{\prime \mu \nu} F_{2}^{\prime}\left(q_{1}^{2}, q_{2}^{2}\right),
$$

with the totally symmetric and mixed (in $\mu, \nu$ ) gauge invariant basis tensors 


$$
\begin{aligned}
& A^{\mu \nu}=Q^{2} P^{\mu} P^{\nu}+P^{2} Q^{\mu} Q^{\nu}-\nu\left(P^{\mu} Q^{\nu}+P^{\nu} Q^{\mu}\right)+\left(\nu^{2}-Q^{2} P^{2}\right) g^{\mu \nu}, \\
& A^{\prime \mu \nu}=-\frac{1}{4} P^{\mu} P^{\nu}+Q^{\mu} Q^{\nu}+\frac{1}{2}\left(P^{\mu} Q^{\nu}-P^{\nu} Q^{\mu}\right)-\left(Q^{2}-\frac{1}{4} P^{2}\right) g^{\mu \nu} .
\end{aligned}
$$

Whereas $A^{\mu \nu}$ has only transverse components, $A^{\prime \mu \nu}$ contributes to both longitudinal and transverse photon helicities:

$$
\begin{aligned}
& T_{\epsilon}^{++}=i\left(\nu^{2}-m_{\epsilon}^{2} Q^{2}\right) F_{\epsilon}\left(q_{1}^{2}, q_{2}^{2}\right)-i\left(Q^{2}-\frac{1}{4} m_{\epsilon}^{2}\right) F_{\epsilon}^{\prime}\left(q_{1}^{2}, q_{2}^{2}\right), \\
& T_{\epsilon}^{00}=i \sqrt{\left(Q^{2}+\frac{1}{4} m_{\epsilon}^{2}\right)^{2}-\nu^{2}} F_{\epsilon}^{\prime}\left(q_{1}^{2}, q_{2}^{2}\right) .
\end{aligned}
$$

Defining the invariant decomposition of the bilocal matrix element on the light cone as

$$
\left\langle\epsilon(P)\left|J_{Q^{2}}^{\sigma}\left(\frac{1}{2} x \mid-\frac{1}{2} x\right)-J_{Q^{2}}^{\sigma}\left(-\frac{1}{2} x \mid \frac{1}{2} x\right)\right| 0\right\rangle=i P^{\sigma} g_{1}(x P)+x^{\sigma} g_{2}(x P),
$$

and introducing the Fourier transform as before, one has

$$
T_{\epsilon}^{\mu \nu} \rightarrow-i\left\{Q^{\mu} P^{\nu}+P^{\mu} Q^{\nu}-\nu g^{\mu \nu}\right\} \frac{1}{Q^{2}} \int_{-1}^{+1} \frac{\mathrm{d} u \psi_{\epsilon}(u)}{1+\xi^{\prime} u}+\text { non-leading gauge terms. }
$$

The expression on the right-hand side can be made explicitly gauge invariant by adding only non-leading terms

$$
T_{\epsilon}^{\mu \nu} \rightarrow i A^{\mu \nu} \frac{1}{\xi^{\prime} Q^{2}} \int_{-1}^{+1} \frac{\mathrm{d} u \psi_{\epsilon}(u)}{1+\xi^{\prime} u}
$$

Taking the antisymmetry of $\psi_{2}(u)$ into account, we get finally

$$
\begin{aligned}
& T_{\epsilon}^{++} \rightarrow-i \xi^{\prime 2} \int_{-1}^{+1} \frac{\mathrm{d} u u \psi_{\epsilon}(u)}{1-\xi^{\prime 2} u^{2}}=i \xi^{\prime 2} \hat{g}_{\epsilon}\left(\xi^{\prime}\right), \\
& T_{\epsilon}^{00} \rightarrow 0 .
\end{aligned}
$$

Because of the antisymmetry of $g_{1}(x P)=-g_{1}(-x P)$, an expression for $\hat{g}_{\epsilon}(0)$ is harder to find than for $\hat{f}_{\pi}(0)$. Expanding the bilocal currents around $x=0$ gives

$$
\left\langle\epsilon(P)\left|J_{Q^{2}}^{\sigma}\left(\frac{1}{2} x \mid-\frac{1}{2} x\right)-J_{Q^{2}}^{\sigma}\left(-\frac{1}{2} x \mid \frac{1}{2} x\right)\right| 0\right\rangle=2 i x_{\rho}\left\langle\epsilon(P)\left|\Theta_{Q^{2}}^{\rho \sigma}(0)\right| 0\right\rangle+\ldots,
$$

where in the quark model

$$
\Theta_{Q^{2}}^{\rho \sigma}(x)=\frac{1}{2} i: \bar{q}(x) \gamma^{\rho} \stackrel{\leftrightarrow}{\partial}^{\sigma} Q^{2} q(x):
$$

is the energy-momentum tensor apart from the matrix $Q^{2}$. From the deep-inelastic electron-proton data, one can conclude that by replacing the singlet piece of $\Theta_{Q^{2}}^{\rho \sigma}$ by the energy-momentum tensor one can get an estimate for matrix elements good to a factor 2 or so [1]. Thus setting $\Theta_{Q^{2}}^{\rho \sigma} \sim \frac{2}{9} \Theta^{\rho \sigma}$ for the matrix element involving the $\mathrm{SU}_{3}$ singlet $\epsilon_{0}$ and defining 


$$
\left\langle\epsilon_{0}(P)\left|\Theta^{\rho \sigma}\right| 0\right\rangle=\frac{1}{3} i f_{\epsilon}\left\{m_{\epsilon}^{2} g^{\rho \sigma}-P^{\rho} P^{\sigma}\right\},
$$

we arrive at

$$
\hat{g}_{\epsilon_{0}}(0)=-\int_{-1}^{+1} \mathrm{~d} u u \psi_{\epsilon_{0}}(u) \sim \frac{8}{27} f_{\epsilon_{0}} .
$$

From phenomenological analyses, several authors have claimed $f_{\epsilon_{0}} \sim f_{\pi}$ (ref. [15]). We therefore expect $\epsilon$ and $\pi$ production to be of the same order of magnitude.

Again applying the quark model to $\hat{g}_{\pi_{N}}, \hat{g}_{\epsilon_{0}}$ and $\hat{g}_{\mathrm{S}} *$ assuming here the identification

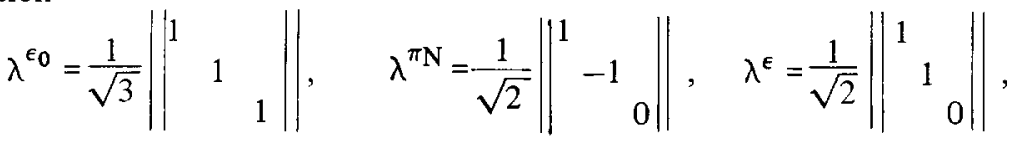

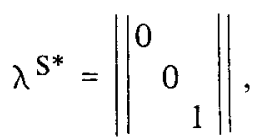

we easily derive for the ratios of the structure functions

$$
\hat{g}_{\epsilon_{0}}\left(\xi^{\prime}\right): \hat{g}_{\pi_{N}}\left(\xi^{\prime}\right): \hat{g}_{\epsilon}\left(\xi^{\prime}\right): \hat{g}_{\mathrm{S}} *\left(\xi^{\prime}\right)=\frac{6}{\sqrt{3}}: \frac{3}{\sqrt{2}}: \frac{5}{\sqrt{2}}: 1 .
$$

Let us emphasize that scalar (and tensor) meson production in $\gamma^{*} \gamma^{*}$ annihilation provides a useful tool to study couplings of the tensor given by (29). From the factor 2 discrepancies mentioned earlier, this is presumably not directly related to the energy-momentum tensor; perhaps it is a piece of that tensor.

Again, we note the dramatic $\xi^{\prime}$ dependence of $W$, which emphasizes the region $\left|\xi^{\prime}\right| \sim \mid$. It is interesting that the $\xi^{\prime 4}$ zero of $W$ as $\xi^{\prime} \rightarrow 0$ is converted to an asymptotic behavior $W \propto\left(Q^{2}\right)^{-2}$ at $q_{1}^{2}=q_{2}^{2}$. Nothing in our analysis (except the precise value of the sum rules) should change in $\gamma^{*}+\gamma^{*} \rightarrow \pi^{+}+\pi^{-}$at threshold, and we conclude that for $q_{1}^{2}=q_{2}^{2} W \propto\left(Q^{2}\right)^{-2}$ here also for $s \simeq 4 m_{\pi}^{2}, q_{1}^{2} \rightarrow-\infty$. Soft-pion techniques can be used to obtain this result as a consequence of the Weinberg sum rules [16]. Here we see that it is a consequence of the quark light-cone algebra or quark parton model. This result does not follow for $j=0$ partons.

\section{3. $A_{1}$ meson}

According to the helicity rules, there are three independent form factors for $A_{1}$ production,

$$
\begin{aligned}
& T_{\mathrm{A}}^{\mu \nu}=i\left(Q e^{*}\right) \epsilon^{\mu \nu \rho \sigma} P_{\rho} Q_{\sigma} F_{\mathrm{A}}\left(q_{1}^{2}, q_{2}^{2}\right)+ \\
& \quad+i\left\{\left(P q_{1}\right) Q^{\mu}-\left(Q q_{1}\right) P^{\mu}\right\} \epsilon^{\nu \rho \sigma \tau} P_{\rho} Q_{\sigma} e_{\tau}^{*} F_{\mathrm{A}}^{\prime}\left(q_{1}^{2}, q_{2}^{2}\right)+ \\
& \quad+i\left\{\left(P q_{2}\right) Q^{\nu}-\left(Q q_{2}\right) P^{\nu}\right\} \epsilon^{\mu \rho \sigma \tau} P_{\rho} Q_{\sigma} e_{\tau}^{*} F_{\mathrm{A}}^{\prime \prime}\left(q_{1}^{2}, q_{2}^{2}\right)
\end{aligned}
$$


The polarization vectors $\epsilon^{(\lambda)}$ are written in the $A_{1}$ rest frame. We have written the decomposition in such a way that the form factors are in a one-to-one correspondence with the helicity amplitudes:

$T_{\mathrm{A}}^{++}=\frac{1}{m_{\mathrm{A}}}\left(\nu^{2}-m_{\mathrm{A}}^{2} Q^{2}\right) F_{\mathrm{A}}\left(q_{1}^{2}, q_{2}^{2}\right)$,

$T_{\mathrm{A}}^{+0}=-\left(\nu^{2}-m_{\mathrm{A}}^{2} Q^{2}\right) \sqrt{-Q^{2}+v-\frac{1}{4} m_{\mathrm{A}}^{2}} F_{\mathrm{A}}^{\prime}\left(q_{1}^{2}, q_{2}^{2}\right)$,

$T_{\mathrm{A}}^{0+}=-\left(\nu^{2}-m_{\mathrm{A}}^{2} Q^{2}\right) \sqrt{-Q^{2}-\nu-\frac{1}{4} m_{\mathrm{A}}^{2}} F_{\mathrm{A}}^{\prime \prime}\left(q_{1}^{2}, q_{2}^{2}\right)$.

The square roots are identical with $\sqrt{-q_{1}^{2}}$ and $\sqrt{-q_{2}^{2}}$, so that the helicity amplitudes for longitudinal photons vanish when the corresponding $q_{i}^{2}=0$. However, $T_{\mathrm{A}}^{++}$also vanishes for both photons on shell, since Bose symmetry requires that $F_{\mathrm{A}}\left(q_{1}^{2}, q_{2}^{2}\right)$ is antisymmetric. Thus the $\mathrm{A}_{1} \gamma \gamma$ coupling vanishes completely for on-shell photons, a well-known fact.

The $\mathrm{L}$ limit in this case is appreciably more complicated than in the preceeding two, not only because of the presence of three form factors, but also because of the need to impose gauge invariance. Inserting the light conc cxpansion into $T_{\mathrm{A}}^{\mu \nu}$, we get

$$
\begin{aligned}
T_{\mathrm{A}}^{\mu \nu} & \rightarrow-i \int \mathrm{d}^{4} x \mathrm{e}^{i Q x}\left[\partial_{\rho} D_{\mathrm{F}}(x)\right]\left\{-i \epsilon^{\mu \rho \nu \sigma}\left\langle A_{1}(P)\left|J_{5 \sigma}^{Q^{2}}\left(\frac{1}{2} x \mid-\frac{1}{2} x\right)+(x \leftrightarrow-x)\right| 0\right\rangle\right. \\
& +s^{\mu \rho \nu \sigma}\left\langle\mathrm{A}_{1}(P)\left|J_{\sigma}^{Q^{2}}\left(\frac{1}{2} x \mid-\frac{1}{2} x\right)-(x \leftrightarrow-x)\right| 0\right\rangle .
\end{aligned}
$$

The invariant decomposition of the bilocal matrix element can be written as

$$
\begin{aligned}
& \left\langle\mathrm{A}_{1}(P)\left|J_{5 \sigma}^{Q^{2}}\left(\frac{1}{2} x \mid-\frac{1}{2} x\right)+(x \leftrightarrow-x)\right| 0\right\rangle=\mathrm{e}_{\sigma}^{*} k_{1}(x P)+i P_{\sigma}\left(e^{*} x\right) k_{2}(x P) \\
& \quad+x_{\sigma}\left(e^{*} x\right) k_{3}(x P) \\
& \left\langle\mathrm{A}_{1}(P)\left|J_{\sigma}^{Q^{2}}\left(\frac{1}{2} x \mid-\frac{1}{2} x\right)-(x \leftrightarrow-x)\right| 0\right\rangle=i \epsilon_{\sigma \alpha \beta \gamma} P^{\alpha} x^{\beta} e^{* \gamma} k^{\prime}(x P) .
\end{aligned}
$$

To begin with, we can easily see that the last term in the axial vector matrix element does not contribute to leading order $\mathrm{O}(1)$ of the helicity amplitude, because $x_{\sigma} \partial_{\rho} x^{-2}$ is symmetrical in the indices $\rho$ and $\sigma$ and thus vanishes when contracted with $\epsilon^{\mu \rho \nu \sigma}$. A somewhat more tedious calculation shows that the vector matrix element does not contribute to leading order either. Inserting the first two terms into the fourier transform, we obtain a term with the structure $\epsilon^{\mu \rho \nu \sigma} e_{\sigma}^{*} Q_{\rho}$. This is not explicitly gauge invariant. However, this tensor can be replaced to leading order by the gauge invariant tensor $\left(Q e^{*}\right) \epsilon^{\mu \rho \nu \sigma} P_{\rho} Q_{\sigma} / \nu$. We are finally left with the representation

$$
\begin{aligned}
T_{\mathrm{A}}^{\mu \nu} & \rightarrow i\left(Q e^{*}\right) \epsilon^{\mu \rho \nu \sigma} P_{\rho} Q_{\sigma}\left\{\frac{1}{\nu Q^{2}} \int_{-1}^{+1} \frac{\mathrm{d} u \chi_{\mathrm{A}}^{1}(u)}{1-\xi^{\prime 2} u^{2}}+\frac{4 \xi^{\prime}}{Q^{4}} \int_{-1}^{+1} \frac{\mathrm{d} u u \chi_{\mathrm{A}}^{2}(u)}{\left(1-\xi^{\prime 2} u^{2}\right)^{2}}\right\}+\ldots \\
& =i\left(Q e^{*}\right) \epsilon^{\mu \rho \nu \sigma} P_{\rho} Q_{\sigma} \frac{1}{\nu Q^{2}}\left\{\hat{\chi}_{\mathrm{A}}^{1}\left(\xi^{\prime}\right)+4 \xi^{\prime 2} \hat{\chi}_{\mathrm{A}}^{2}\left(\xi^{\prime}\right)\right\}
\end{aligned}
$$


and we get, as expected, a finite contribution only to $T_{\mathrm{A}}^{++}$,

$$
T_{\mathrm{A}}^{++} \rightarrow \frac{1}{m_{\mathrm{A}}} \xi^{\prime}\left\{\chi_{\mathrm{A}}^{1}\left(\xi^{\prime}\right)+4 \xi^{\prime 2} \chi_{\mathrm{A}}^{2}\left(\xi^{\prime}\right)\right\} .
$$

$\Lambda$ gain, we can relate the scaling function $\hat{\chi}_{A}^{1}\left(\xi^{\prime}\right)$ at $\xi^{\prime}=0$ to the coupling constant $f_{\mathrm{A}}$ defined by $\left\langle\mathrm{A}_{1}\left|J_{5 \sigma}\right| 0\right\rangle=e_{\sigma}^{*} f_{\mathrm{A}}$. We get

$$
\hat{\chi}_{\mathrm{A}}^{1}(0)=\int_{-1}^{+1} \mathrm{~d} u \chi_{\mathrm{A}}^{1}(u)=\frac{2}{3} f_{\mathrm{A}} \text {. }
$$

The scaling functions of the other $1^{++}$particles within the same fashion as before lated in the same fashion as before to $\hat{\chi}_{A}^{1,2}\left(\xi^{\prime}\right)$; because of the present dubious experimental status of these particles, we will not dwell on this extension.

\section{4. f-meson}

The last resonances we shall discuss here are the $2^{++}$particles $f, f^{\prime}$ and $\mathrm{A}_{2}^{0}$. This case is the most complicated one because of the presence of five form factors. However, we shall confine ourselves to the discussion of the helicity amplitudes $T_{\mathrm{f}}^{++}$and $T_{\mathrm{f}}^{+-}$, arguing that $T_{\mathrm{f}}^{00}, T_{\mathrm{f}}^{+0}$ and $T_{\mathfrak{f}}^{0+}$ vanish in the $\mathrm{L}$ limit we are interested in. This follows most simply by noting that the leading contributions come from the vector bilocal and that they are symmetric under interchange of $\mu$ and $\nu$ (the tensor indices of the currents). The only such tensors with this property are also fully transverse. They are*

$$
\begin{aligned}
& B_{1}^{\mu \nu}=\left\{Q^{2} P^{\mu} P^{\nu}+P^{2} Q^{\mu} Q^{\nu}-\nu\left(P^{\mu} Q^{\nu}+P^{\nu} Q^{\mu}\right)+\left(\nu^{2}-P^{2} Q^{2}\right) g^{\mu \nu}\right\}\left(Q^{\alpha} Q^{\beta} E_{\alpha \beta}^{*}\right) \\
& B_{2}^{\mu \nu}=\left\{P^{\mu} P^{\nu}-P^{2} g^{\mu \nu}\right\}\left(Q^{\alpha} Q^{\beta} E_{\alpha \beta}^{*}\right)+\left(\nu^{2}-P^{2} Q^{2}\right) E^{* \mu \nu} \\
& \quad+\left\{-\nu\left(P^{\mu} E_{\beta}^{* \nu}+P^{\nu} E_{\beta}^{* \mu}\right)+P^{2}\left(Q^{\mu} E_{\beta}^{* \nu}+Q^{\nu} E_{\beta}^{* \mu}\right)\right\} Q^{\beta} .
\end{aligned}
$$

where $E^{\mu \nu}$ is the f-meson polarization tensor $\left(E_{\mu}^{\mu}=0, E^{\mu \nu}=E^{\nu \mu}, E^{\mu \nu} P_{\nu}=0\right)$; a representation in the f-meson rest frame is given by $E_{\left(\lambda+\lambda^{\prime}\right)}=e_{(\lambda)}^{*} e_{\left(\lambda^{\prime}\right)}$. The transverse form factors, defined by

$$
T_{\mathrm{f}}^{\mu \nu}=i B_{1}^{\mu \nu} F_{\mathrm{f}}^{1}\left(q_{1}^{2}, q_{2}^{2}\right)+i B_{2}^{\mu \nu} F_{\mathrm{f}}^{2}\left(q_{1}^{2}, q_{2}^{2}\right)+\ldots,
$$

are related to the helicity amplitudes by

$$
\begin{aligned}
& T_{\mathrm{f}}^{++}=\frac{2 i}{\sqrt{6}} \frac{1}{m_{\mathrm{f}}^{2}}\left(\nu^{2}-m_{\mathrm{f}}^{2} Q^{2}\right)^{2} F_{\mathrm{f}}^{1}\left(q_{1}^{2}, q_{2}^{2}\right)-\frac{i}{\sqrt{6}}\left(\nu^{2}-m_{\mathrm{f}}^{2} Q^{2}\right) F_{\mathrm{f}}^{2}\left(q_{1}^{2}, q_{2}^{2}\right)+\ldots, \\
& T_{\mathrm{f}}^{+-}=i\left(\nu^{2}-m_{\mathrm{f}}^{2} Q^{2}\right) F_{\mathrm{f}}^{2}\left(q_{1}^{2}, q_{2}^{2}\right)+\ldots .
\end{aligned}
$$

In order to discuss the $\mathrm{L}$ limit, we insert the light cone expansion into $T_{\mathrm{f}}^{\mu \nu}$, ending with something analogous to that for the $\mathrm{A}_{1}$ case. The invariant decomposition of the bilocal matrix element is

* The complete basis is given in the appendix. 


$$
\begin{aligned}
& \left\langle f(P)\left|J_{\sigma}^{Q^{2}}\left(\frac{1}{2} x \mid-\frac{1}{2} x\right)-(x \leftrightarrow-x)\right| 0\right\rangle=E^{* \alpha \beta}\left\{x_{\alpha} g_{\sigma \beta} k_{1}(x P)\right. \\
& \left.\quad+i x_{\alpha} x_{\beta} P_{\sigma} k_{2}(x P)+x_{\alpha} x_{\beta} x_{\sigma} k_{3}(x P)\right\}, \\
& \left\langle f(P)\left|J_{5 \sigma}^{Q^{2}}\left(\frac{1}{2} x \mid-\frac{1}{2} x\right)+(x \leftrightarrow-x)\right| 0\right\rangle=i E^{* \alpha \beta} x_{\alpha} \epsilon_{\sigma \beta \gamma \sigma} x^{\gamma} P^{\sigma} k^{\prime}(x P) .
\end{aligned}
$$

As before, the axial vector term and the trilinear term of the vector bilocal piece do not enter the asymptotic behavior of the form factors. The contribution of $k_{1}$ and $k_{2}$ is

$$
\begin{aligned}
& T_{\mathrm{f}}^{\mu \nu} \rightarrow 2 i B_{1}^{\mu \nu}\left(\frac{1}{\nu^{2} Q^{4}} \int_{-1}^{+1} \frac{\mathrm{d} u \rho_{\mathrm{f}}^{1}(u)}{\left(1+u \xi^{\prime}\right)^{2}}-\frac{4}{\nu Q^{6}} \int_{-1}^{+1} \frac{\mathrm{d} u \rho_{\mathrm{f}}^{2}(u)}{\left(1+u \xi^{\prime}\right)^{3}}\right) \\
& \quad+2 i B_{2}^{\mu \nu} \frac{1}{\nu^{2} Q^{2}} \int_{-1}^{+1} \frac{\mathrm{d} u \rho_{\mathrm{f}}^{1}(u)}{1+u \xi^{\prime}}
\end{aligned}
$$

Notice that the gauge problems appear only in longitudinal pieces. However, as long as the components $T_{\mathrm{f}}^{\mu \nu}$ stay finite (which is so in our case) the helicity amplitudes $T^{0 \pm}$ and $T^{00}$ vanish as $1 / \sqrt{ }-Q^{2}$ and $1 /\left(-Q^{2}\right)$ respectively. The remaining transverse amplitudes are

$$
\begin{aligned}
& T_{\mathrm{f}}^{++} \rightarrow \frac{2 i}{\sqrt{6}} \frac{1}{m_{\mathrm{f}}^{2}} \xi^{\prime 2}\left\{\int_{-1}^{+1} \frac{\mathrm{d} u \rho_{\mathrm{f}}^{1}(u)}{\left(1+u \xi^{\prime}\right)^{2}}-4 \xi^{\prime} \int_{-1}^{+1} \frac{\mathrm{d} u \rho_{\mathrm{f}}^{2}(u)}{\left(1+u \xi^{\prime}\right)^{3}}\right\}, \\
& T_{\mathrm{f}}^{+-} \rightarrow 0,
\end{aligned}
$$

and we have the interesting result that the tensor mesons are produced only in states of helicity zero. The vanishing of the amplitudes with a longitudinal photon and the predominant zero helicity of the produced meson can be checked both in the lepton distributions for $e^{-}+e^{ \pm} \rightarrow e^{-}+e^{ \pm}+f$ as well as in the $f \rightarrow \pi+\pi$ decay distributions.

In order to show that $T_{\mathrm{f}}^{++}$is non vanishing, we derive a sum rule involving the zeroth moment of the structure function $\rho_{\mathrm{f}}^{1}$. Returning to the expansion of $J^{\sigma}\left(\frac{1}{2} x \mid-\frac{1}{2} x\right)$, we see that we have to determine $x_{\rho}\left\langle f\left|\Theta_{Q^{2 g}}^{\rho \sigma}\right| 0\right\rangle$. We can try to estimate the magnitude of such a matrix element between the vacuum and an SU(3) singlet state by assuming it to be of the same order of magnitude as the matrix element involving the energy momentum tensor. The identification should be sufficient for a factor-of-two estimate, to judge by the approximate validity of the Mack sum rule for the nucleon structure functions in inelastic electron-nucleon scattering [1]. Anyway, defining for an $\mathrm{SU}_{3}$ singlet $\mathrm{f}^{(0)}$

$$
\left\langle\mathrm{f}^{(0)}\left|\Theta^{\rho \sigma}\right| 0\right\rangle=i f_{\mathrm{f}} E^{* \rho \sigma},
$$

we get the sum rule

$$
\int_{-1}^{+1} \mathrm{~d} u \rho_{\mathrm{f}}^{1}(u) \sim-\frac{1}{9} f_{\mathrm{f}},
$$


where the quantity is to be estimated by using tensor meson dominance not for $\Theta_{O^{2}}^{\rho \sigma}$ but for the energy momentum tensor. Writing $\left\langle\pi\left|\Theta \Theta^{\rho \sigma}\right| \pi\right\rangle=2 P^{\rho} P^{\sigma}$, we immediately get the estimate

$$
f_{\mathrm{f}} \sim m_{\mathrm{f}}^{2} / g_{\mathrm{f} \pi \pi}
$$

for $f_{\mathrm{f}}$ (ref. [17]). The relations between the scaling functions of the various $2^{++}$ nonet members should be identical to those of the (ideally mixed) $0^{++}$mesons.

We have some general comments on the $\gamma^{*} \gamma^{*}$-meson form factors.

(i) The preceeding arguments can be generalized to include higher spin resonances, and we expect $T^{00}, T^{+0}, T^{0+}, T^{+-} \rightarrow 0$, generally. The vanishing of the first three helicity amplitudes can be obtained under very weak assumptions, and we have already referred to this. Since we have finite components of the tensor $T^{\mu \nu}$ in the $\mathrm{L}$ limit as can be verified from our examples we can, by applying gauge invariance $q_{1 \mu} T^{\mu \nu}=q_{2 \nu} T^{\mu \nu}=0$, obtain the result that

$$
\begin{aligned}
& T^{0 \pm}=\frac{m}{\sqrt{-q_{1}^{2}}}\left[\left(1-Q^{2} / \nu\right)+\ldots\right] T^{3 \pm}=\mathrm{O}\left(1 / \sqrt{-Q^{2}}\right) T^{3 \pm}=\mathrm{O}\left(1 / \sqrt{-Q^{2}}\right), \\
& T^{00}=\frac{m^{2}}{\sqrt{q_{1}^{2} q_{2}^{2}}}\left[\left(1-Q^{4} / \nu^{2}\right)+\ldots\right] T^{33}=\mathrm{O}\left(1 /\left(-Q^{2}\right)\right) T^{33}=\mathrm{O}\left(1 /\left(-Q^{2}\right)\right) .
\end{aligned}
$$

The reason why $T^{+-} \rightarrow 0$ is a bit more obscure, but we shall see in the next section that to leading order in the $\mathrm{L}$ limit, two photons with parallel spins cannot couple to a massless free fermion-antifermion pair.

(ii) The smooth threshold assumption is an even stronger constraint for high-spin mesons than in the $\pi^{0}$ and $\epsilon$ cases; for the $\mathrm{f}, \rho_{\mathrm{f}}^{1}$ and $\rho_{\mathrm{f}}^{2}$ must vanish at least as fast as $(1-u)^{2}$ and $(1-u)^{3}$ for $u \rightarrow 1$. If the structure functions are to be reasonably smooth in $\xi$, the threshold behaviour must be even stronger. This is a reflection of an anogalous situation in the vertex $\gamma^{*} \rho^{0} \mathrm{f}^{0}$ which is vaguely similar to $\gamma^{*} \gamma^{*} \mathrm{f}^{0}$ at $\xi= \pm 1$. Unless the invariant amplitudes decrease rapidly as $q^{2} \rightarrow \infty$, cross sections like $\sigma\left(\mathrm{e}^{+} \mathrm{e}^{-} \rightarrow \gamma^{*}(q) \rightarrow \rho^{0} \mathrm{f}^{0}\right)$ could have unreasonable high-energy behaviour, the invariant amplitudes being multiplied by kinematical factors which blow up as $q^{2} \rightarrow \infty$.

(iii) If the smooth threshold assumption is really valid, one can check some of thes? results in $\gamma^{*}+\gamma \rightarrow$ mesons, where one photon is nearly real [18]. The most striking fact is that the tensor mesons should be produced in states with zero helicity in the $\gamma^{*} \gamma$ c.m. frame. If the f-meson, however, is produced with helicity \pm 2 in real $\gamma \gamma$ collisions (as some authors have claimed [19]), there should be a rapid variation of the $f \rightarrow \pi+\pi$ decay distributions as a function of $q^{2}$ of one of the photons as it increases from zero to the region where the scaling behavior should hold.

(iv) We remark further that the presence of a light cone term when one photon has $q_{1}^{2}=0$ results in a slower decrease of the $\gamma^{*} \gamma$-meson form factor than one 
would expect for the $\gamma^{*} \rho$-meson form factor following arguments of Brandt and Preparata (see also Wilson [20]). This means that there is no compelling reason why the threshold behavior for inelastic electron-photon scattering should resemble that for inelastic electron-rho meson scattering. This remark is not frivolous, since the related processes $\mathrm{e}^{+} \mathrm{e}^{-} \rightarrow \gamma^{*} \rightarrow\left(\rho^{0}, \gamma\right)+\mathrm{X}$ should be measurable and a comparison possible in principle.

(v) The results obtained so far are based on the connected part of the light cone algebra and are probably quite general, perhaps even true. It is interesting, however, that the structure of the absorptive part of $\gamma^{*} \gamma^{*} \rightarrow \gamma^{*} \gamma^{*}$ obtained in this way in the resonance region is the same as that following from the disconnected part of the light cone algebra at large $s$. If one accepts this latter prediction, the possibility arises that this disconnected part in fact just average the contribution of the connected parts and should not be added to it as dutiful followers of the parton model would do. This possibility would amount to a kind of duality for the light cone algebra, for which the usual parton model prescription would amount to double counting. A realization of this would probably make sense only in the context of the "connected" models we mentioned earlier. For the purposes of this paper, we are taking the more conventional view that the connected and disconnected pieces should be added, as is the case in the Landshoff, Polkinghorne, Short model, and as one would (actually equivalently) infer from the distinct character of the connected and disconnected pieces of the light cone algebra.

\section{The continuum region}

Increasing $s$ in the asymptotic region of $q_{1}^{2}$ and $q_{2}^{2}$, it is natural to ask about the behavior of the absorptive part of the amplitude $\gamma^{*} \gamma^{*} \rightarrow \gamma^{*} \gamma^{*}$ above the reso. nance region. More generally, one can consider the $S$ limit where $s \rightarrow \infty$ with $\xi=$ $=\left(q_{2}^{2}-q_{1}^{2}\right) /\left(q_{2}^{2}+q_{1}^{2}\right)$ and $\omega=1-s /\left(q_{2}^{2}+q_{1}^{2}\right)$ fixed. The limit just mentioned (the DL limit) then just corresponds to threshold, $\omega \rightarrow 1$ at fixed $\xi$.

In the model of Landshoff, Polkinghorne and Short, the leading term in the S limit is given by the disconnected diagram of fig. $4 \mathrm{~d}$ and its crossed diagram. This is a consequence of the cutoff in the model and is presumably a general feature of this sort of model. A nice virtue of this is that we can calculate the absorptive part of $\gamma^{*} \gamma^{*} \rightarrow \gamma^{*} \gamma^{*}$ exactly in terms of the quark charges. We have only to calculate the helicity amplitudes in massless spinor electrodynamics for the box graph contribution to $\gamma^{*} \gamma^{*} \rightarrow \gamma^{*}$. This yields, in the S limit,

$$
W^{++ \pm \pm}=\sum_{i} e_{i}^{4} \int_{-1}^{+1} \frac{\mathrm{d} z}{2 \pi} \frac{\left(1-z^{2}\right)\left\{\left(1-\omega-\xi^{2}\right)^{2} z^{2} \pm \omega^{2} \xi^{2}\right\}}{\left\{\omega^{2}+z^{2}\left(1-\omega^{2}-\xi^{2}\right)\right\}^{2}} \text {, }
$$




$$
\begin{aligned}
& W^{+-+-}=\sum_{i} e_{i}^{4} \int_{-1}^{+1} \frac{\mathrm{d} z}{2 \pi} \cdot \frac{\left(1-z^{4}\right) \omega^{2}(1-\omega)^{2}}{\left\{\omega^{2}+z^{2}\left(1-\omega^{2}-\xi^{2}\right)\right\}^{2}}, \\
& W^{0000}=\sum_{i} e_{i}^{4} 4(\omega-1)^{2}\left(1-\xi^{2}\right) \int_{-1}^{+1} \frac{\mathrm{d} z}{2 \pi} \frac{z^{2}\left(1-z^{2}\right)}{\left\{\omega^{2}+z^{2}\left(1-\omega^{2}-\xi^{2}\right)\right\}^{2}}, \\
& W^{++00}=\sum_{i} e_{i}^{4} 2(\omega-1)\left(\xi^{2}+\omega-1\right) \sqrt{1-\xi^{2}} \int_{-1}^{+1} \frac{\mathrm{d} z}{2 \pi} \frac{z^{2}\left(1-z^{2}\right)}{\left\{\omega^{2}+z^{2}\left(1-\omega^{2}-\xi^{2}\right)\right\}^{2}}, \\
& W^{+00-}=\sum_{i} e_{i}^{4}(1-\omega) \sqrt{1-\xi^{2}} \int_{-1}^{+1} \frac{\mathrm{d} z}{2 \pi} \\
& \times \frac{\left(\xi^{2}-1\right) z^{2}\left(1+z^{2}\right)+\omega\left(z^{2}-1\right)\left[8 z^{2}+\omega\left(1-z^{2}\right)\right]}{\left\{\omega^{2}+z^{2}\left(1-\omega^{2}-\xi^{2}\right)\right\}^{2}} \\
& W^{+0+0}=\sum_{i} e_{i}^{4}(\omega-1)(1 \mp \xi) \int_{-1}^{+1} \frac{\mathrm{d} z}{2 \pi} \frac{Y\left(\omega, \pm \xi ; z^{2}\right)}{\left\{\omega^{2}+z^{2}\left(1-\omega^{2}-\xi^{2}\right)\right\}^{2}} \text {, }
\end{aligned}
$$

with

$$
\begin{aligned}
& Y\left(\omega, \pm \xi ; z^{2}\right)=z^{2}\left(1+z^{2}\right)\left\{2(1-\omega)(1 \pm \xi)+\left(\xi^{2}+\omega^{2}-1\right)\right\}+ \\
& \quad+z^{2}\left\{4 \omega(1-\omega \pm \xi)+\omega^{2}\right\}+\omega^{2} .
\end{aligned}
$$

Apparently, we express this result in a compact form by defining the ratios [21]

$$
\begin{gathered}
T=\lim _{s \rightarrow \infty} \frac{\sigma_{\gamma \gamma^{+}}(\mathrm{ee} \rightarrow \mathrm{eeX})}{\sigma_{\gamma \gamma^{+}}(\mathrm{e} \rightarrow \mathrm{e} \mu \bar{\mu})}=\sum_{i} e_{i}^{4}, \\
R=\lim _{q^{2} \rightarrow \infty} \frac{\sigma_{\gamma}(\overline{\mathrm{e}} \rightarrow \mathrm{X})}{\sigma_{\gamma}(\mathrm{e} \overline{\mathrm{e}} \rightarrow \mu \bar{\mu})}=\sum_{i} e_{i}^{2},
\end{gathered}
$$

where the subscript " $\gamma \gamma+$ ". means that the one-photon background to the process has been subtracted; hence the final state has positive charge conjugation. There is an appreciable advantage to measuring these ratios experimentally, since one can check $T$ and $R$ simply by taking cross-section ratios. Note that if one does not subtract the one-photon background to $\sigma_{\gamma \gamma}(\mathrm{ee} \rightarrow \mathrm{eeX})$, the ratio $\sigma_{\gamma \gamma}(\mathrm{X}) / \sigma_{\gamma \gamma}(\mu \bar{\mu})$ is

$$
\frac{\sigma_{\gamma \gamma}(\mathrm{ee} \rightarrow \mathrm{eeX})}{\sigma_{\gamma \gamma}(\mathrm{ee} \rightarrow \mathrm{ee} \mu \bar{\mu})}=\frac{T+\beta R}{1+\beta} \text { with } \beta=\frac{\sigma_{\gamma \gamma}(\mathrm{ee} \rightarrow \mathrm{ee} \mu \bar{\mu} ; C(\mu \bar{\mu})=-1)}{\sigma_{\gamma \gamma}(\mathrm{ee} \rightarrow \operatorname{ee} \mu \bar{\mu} ; C(\mu \bar{\mu})=+1)} \text {, }
$$

where $\beta$ can be calculated from QED (the above ratio is $R$ in the Han-Nambu model where $T=R$ ).

The detailed structure of the helicity amplitudes contains much more informa: tion. 
(i) The threshold limit $\omega \rightarrow 1$ leads to the light cone algebra result that the only non-zero amplitudes are [6], [10]:

$$
W^{++ \pm \pm}=\sum_{i} e_{i}^{4} \xi^{2} \int_{-1}^{+1} \frac{\mathrm{d} z}{2 \pi} \frac{1-z^{2}}{\left(z^{2} \xi^{2}-1\right)^{2}}\left(z^{2} \xi^{2} \pm 1\right),
$$

which exhibits the striking similarity to the kinematic structure of the resonance amplitudes which we emphasized in the last section. The contributions from states of definite $s$-channel parity $( \pm)$ are [6],

$$
\frac{1}{2}\left(W^{++++} \pm W^{++--}\right)=\sum_{i} e_{i}^{4} \xi^{2} \int_{-1}^{+1} \frac{\mathrm{d} z}{2 \pi} \frac{\left(1-z^{2}\right)}{\left(1-z^{2} \xi^{2}\right)^{2}}\left\{\begin{array}{c}
z^{2} \xi^{2} \\
1
\end{array}\right\} \text {. }
$$

The light cone structure near $\omega \rightarrow 1$ might be of more general validity than the ex pressions for the helicity amplitudes in the $\mathrm{S}$ limit, which depend to a weak extent on models (namely, on the existence of a softening or a transverse momentum cut off).

(ii) The longitudinal amplitudes survive in the S-limit because of the absence of a cut-off in the surviving diagram. These longitudinal pieces are not very big, since they are constrained to vanish for $\omega \rightarrow \infty$ and $\omega \rightarrow 1$.

(iii) The limit $\xi^{2} \rightarrow 1$ of the helicity amplitudes in the S limit is logarithmically divergent. There are two arguments why this could carry over into a log divergence of the structure functions for inelastic electron-photon scattering [18], which is formally a different limit $\left(q_{1}^{2}=0\right.$, with $s / q_{2}^{2}$ fixed). First, evidence from deep inelastic electron-rucleon scattering leads one to the inference that scaling limits are reached rapidly and that in fact the limits $s \rightarrow \infty, q_{1}^{2}$ fixed and $s \rightarrow \infty, s / q_{1}^{2}$ fixed are interchangeable in the sence that the former one corresponds to $s / q_{1}^{2} \rightarrow \infty$ in the latter. Our example here is just an extension of this point. Second, in the LPS model this disconnected diagram (4d) appears independent of the kinematic variables and leads directly to $\log$ divergent structure functions for real photons or a log divergence when $q_{2}^{2} \rightarrow-\infty$ and $q_{1}^{2}$ fixed. In fact, one expects a logarithmic divergence for any limit where the ratio $q_{2}^{2} / q_{1}^{2}$ increases without bound [22].

So far, we have concerned ourselves mostly with the class of limits where $q_{1}^{2}$ and $q_{2}^{2}$ increase as fast or faster than the squared c.m. energy $s$. We have given cross section estimates for this process elsewhere [6], and shall not discuss this point further here. The reason for our concentration on this limit is the wealth of explicit predictions we can obtain from light cone or parton model ideas. The question arises whether one can say anything about those limits where $s$ increases faster than $q_{1}^{2}$ and $q_{2}^{2}$ as all tend to infinity. This is certainly of practical relevance, as one has to test our predictions at finite values of the invariants, and not in formal limits. The presence of large contributions from such "scaling Regge" amplitudes for a big enough range of $s$ and $q_{1}^{2}$ and $q_{2}^{2}$ could vitiate the hope of testing light cone algebra and parton model predictions. More over, these contributions (fig. 4a) are of interest in their own right. We shall at tempt here to speculate on their behavior. From 
factorization and the smallness of the ratio $R=\sigma_{\mathrm{L}} / \sigma_{\mathrm{T}}$ in inelastic electron-nucleon scattering, we conclude that the dominant amplitude for $\gamma^{*} \gamma^{*} \rightarrow \gamma^{*} \gamma^{*}$ in the limit where $q_{1}^{2} \rightarrow-\infty, q_{2}^{2} \rightarrow-\infty$ and $s / q_{1}^{2} q_{2}^{2} \rightarrow \infty$ is

$$
W_{R}^{++++}-w_{R}^{+++-}=\sum_{R} \bar{\beta}_{R}\left(\frac{s s_{0}}{\left(q_{1}^{2}-m_{0}^{2}\right)\left(q_{2}^{2}-m_{0}^{2}\right)}\right)^{\alpha_{R}} \text {. }
$$

Note that whereas we had in the S limit dimensionless functions which were constants at fixed $\xi$ and $\omega$, we have here helicity amplitudes which increase without bound in the mentioned limit. As an estimate, we choose $m_{0}^{2} \simeq 0.2 \mathrm{GeV}^{2}$ from parametrizations of $\nu W_{2}\left(\nu, q^{2}\right)$ in eN scattering, $s_{0} \simeq 1 \mathrm{GeV}^{2}$ and $\bar{\beta}_{R}$ from factorization; for convenience we ignore everything but the pomeron. In the region of small scattering but angle $\left|q_{1}^{2}\right|,\left|q_{2}^{2}\right| \gtrsim 0.3 \mathrm{GeV}^{2}$ we can write down an estimate for the cross section $\mathrm{e}^{+}+\mathrm{e}^{-} \rightarrow \mathrm{e}^{+}+\mathrm{e}^{-}+\mathrm{X}$ on the same basis as the WeiszäckerWilliams estimates in the region $q_{i}^{2} \sim-m_{\mathrm{e}}^{2}$. The familiar $\log E / m_{\mathrm{e}}$ factors are missing and we find as an estimate $\sigma \sim(\alpha / \pi)^{2} \sigma \gamma^{*} \gamma^{*} \sim 10^{-36} \mathrm{~cm}^{2}$. Thus contributions of this sort are expected to be about the same order as the light cone terms, and not very much bigger.

From the fact that the comected anplitudes in electron-nucleon scattering decrease rapidly as $\left|q^{2}\right| \gg s$ (in the LPS model this is a consequence of the softening and of the $t_{\min } \propto 1 /(1-\omega)$ effect), we infer that here also the connected amplitudes should decrease as fast as faster than a power of $q_{1}^{2} q_{2}^{2} / \mathrm{s}^{2}$. In inelastic electron-nucleon scattering this threshold behavior of the connected amplitude sets in already for $\omega^{\prime}=1+s /\left(-q^{2}\right)<3$ or $\left|q^{2}\right| \gtrsim \frac{1}{2} s$, consistent with the $t_{\min }$ effect expextations. It will be interesting to see if an analogous behavior can be observed in $\gamma \gamma$ annihila. tion. The practical test of light cone algebra predictions above the resonance region in $s$ evidently depends on the behavior of the Regge terms in this limit. This question needs a more detailed analysis.

\section{Conclusion}

In the forgoing sections we have concentrated on tests of ideas from the quark light cone algebra and related parton model ideas. in processes where two virtual photons annihilate into hadrons. For the time being processes of this kind offer the only possibility of checking the light cone algebra for non-diagonal matrix elements and also for commutators of bilocal currents among themselves. Because of this, we have first studied the production of resonances with low mass by colliding photon beams, where the photons have large virtual mass $\left(\gamma^{*}+\gamma^{*} \rightarrow\right.$ resonance). We have calculated the asymptotic behavior of the corresponding form factors and the absolute scale of the amplitudes. Both are determined by the light cone algebra. As a consequence, rather detailed predictions emerge for production of even charge conjugation states is electron-electron collisions. In a second step, we have studied hadron production in the continuum region above the prominent resonances. In 
that region. the bilocal algebra predicts the amplitudes for $\mathrm{e}^{-}+\mathrm{e}^{ \pm} \rightarrow \mathrm{e}^{-}+\mathrm{e}^{ \pm}+\mathrm{X}$ to be of the same structure as $\mathrm{e}^{-}+\mathrm{e}^{ \pm} \rightarrow \mathrm{e}^{-}+\mathrm{e}^{ \pm}+\mu \bar{\mu}$ (confining ourselves to even charge conjugation states in an appropriately defined kinematical region). This picture is similar to that for one photon $\mathrm{e}^{+} \mathrm{e}^{-}$annihilation where one predicts $\sigma\left(\mathrm{e}^{+} \mathrm{e} \rightarrow \mathrm{X}\right) / \sigma\left(\mathrm{e}^{+} \mathrm{e}^{--} \rightarrow \mu^{+} \mu\right)=$ constant. Because we are dealing with spacelike photons, there is a good chance to observe this scaling behavior even for fairly low photon masses, as we have learned from deep inelastic electron-nucleon scattering. We hope that the appropriate experiments will be attempted with the colliding beam machines now coming into operation. Even the experimental proof that such processes exist would yield strong support to light cone and parton model ideas: pointlike couplings give large cross sections, which are pushed to lower values if decreasing form factors are included in this process with four currents. We believe that it is possible to gain a deep insight into the structure of the electromagnetic current by studying such processes.

\section{Appendix}

The complete set of basis tensors for the $f \gamma^{*} \gamma^{*}$ vertex is

$$
T_{\mathrm{f}}^{\mu \nu}=i \sum_{k=1}^{5} B_{k}^{\mu \nu} F_{k}^{\mathrm{f}}\left(q_{1}^{2}, q_{2}^{2}\right) \text {, }
$$

with

$$
\begin{aligned}
& B_{1}^{\mu \nu}=\left\{Q^{2} P^{\mu} P^{\nu}+P^{2} Q^{\mu} Q^{\nu}-\nu\left[P^{\mu} Q^{\nu}+P^{\nu} Q^{\mu}\right]+\left[\nu^{2}-P^{2} Q^{2}\right] g^{\mu \nu}\right\}\left(Q^{\alpha} Q^{\beta} E_{\alpha \beta}^{*}\right) . \\
& B_{2}^{\mu \nu}=\left\{P^{\mu} P^{\nu}-P^{2} g^{\mu \nu}\right\}\left(Q^{\alpha} Q^{\beta} E_{\alpha \beta}^{*}\right)+\left[\nu^{2}-P^{2} Q^{2}\right] E^{* \mu \nu} \\
& +\left\{-\nu\left[P^{\mu} E_{\beta}^{* \nu}+P^{\nu} E_{\beta}^{* \mu}\right]+P^{2}\left[Q^{\mu} E_{\beta}^{* \nu}+Q^{\nu} E_{\beta}^{* \mu}\right]\right\} Q^{\beta}, \\
& B_{3}^{\mu \nu}=\left\{-\frac{1}{4} P^{\mu} P^{\nu}+Q^{\mu} Q^{\nu}+\frac{1}{2}\left[P^{\mu} Q^{\nu}-P^{\nu} Q^{\mu}\right]-\left[Q^{2}-\frac{1}{4} P^{2}\right] g^{\mu \nu}\right\}\left(Q^{\alpha} Q^{\beta} E_{\alpha \beta}^{*}\right), \\
& B_{4}^{\mu \nu}=\left\{-P^{\mu} P^{\nu}+P^{2} g^{\mu \nu}\right\} Q^{2}\left(Q^{\alpha} Q^{\beta} E_{\alpha \beta}^{*}\right)-P^{2} Q^{\mu} Q^{\nu}\left(Q^{\alpha} Q^{\beta} E_{\alpha \beta}^{*}\right) \\
& +\nu^{2}\left(\frac{1}{4} P^{2}-Q^{2}\right) E^{* \mu \nu}+\left\{\nu Q^{2}\left[P^{\mu} E_{\beta}^{* \nu}+P^{\nu} E_{\beta}^{* \mu}\right]\right. \\
& \left.+\frac{1}{2} P^{2}\left[Q^{\mu} E_{\beta}^{* \nu}-Q^{\nu} E_{\beta}^{* \mu}\right]\right\} Q^{\beta} \text {, } \\
& B_{5}^{\mu \nu}=P^{2} g^{\mu \nu}\left(Q^{\alpha} Q^{\beta} E_{\alpha \beta}^{*}\right)-P^{2}\left(\frac{1}{4} P^{2}-Q^{2}\right) E^{* \mu \nu} \\
& \left\{\frac{1}{2} P^{2}\left[P^{\mu} E_{\beta}^{* \nu}-P^{\nu} E_{\beta}^{*}{ }_{\beta}^{\mu}\right]+P^{2}\left[Q^{\mu} E_{\beta}^{* \nu}+Q^{\nu} E_{\beta}^{* \mu}\right]\right\} Q^{\beta} .
\end{aligned}
$$


Then the helicity form factor is

$$
\begin{aligned}
T_{\mathrm{f}}^{\lambda_{1} \lambda_{2}} & =\epsilon_{\mu}^{\left(\lambda_{1}\right)} \epsilon_{\nu}^{\left(\lambda_{2}\right)} T_{\mathrm{f}}^{\mu \nu} \\
& =i \sum_{k=1}^{5} B_{k}^{\lambda_{1} \lambda_{2}} F_{k}^{\mathrm{f}}\left(q_{1}^{2}, q_{2}^{2}\right) .
\end{aligned}
$$

The various contributions $B_{i}^{\lambda_{1} \lambda_{2}}$ to the helicity form factors are

$$
\begin{aligned}
T_{\mathrm{f}}^{++}: B_{1}^{++} & =\frac{2}{\sqrt{6}} \frac{1}{P^{2}}\left[\nu^{2}-P^{2} Q^{2}\right]^{2}, \quad B_{2}^{++}=-\frac{1}{\sqrt{6}}\left[\nu^{2}-P^{2} Q^{2}\right], \\
B_{3}^{++} & =\frac{2}{\sqrt{6}}\left[\frac{1}{4} P^{2}-Q^{2}\right] \frac{1}{P^{2}}\left[\nu^{2}-P^{2} Q^{2}\right] \\
B_{4}^{++} & =\frac{1}{\sqrt{6}}\left[\nu^{2}\left(\frac{1}{4} P^{2}+Q^{2}\right)-2 P^{2} Q^{4}\right] \\
B_{5}^{++} & =\frac{1}{\sqrt{6}}\left[2 \nu^{2}-P^{2}\left(\frac{1}{4} P^{2}+Q^{2}\right)\right] \\
T_{\mathrm{f}}^{+-}: B_{1}^{+-} & =0, \quad B_{2}^{+-}=\left[\nu^{2}-P^{2} Q^{2}\right] \\
B_{3}^{+-} & =0, \quad B_{4}^{+-}=\nu^{2}\left[\frac{1}{4} P^{2}-Q^{2}\right] \\
B_{5}^{+-} & =-P^{2}\left[\frac{1}{4} P^{2}-Q^{2}\right] . \\
T_{\mathrm{f}}^{00}: B_{1}^{00} & =0, \quad B_{2}^{0+}=0, \\
T_{\mathrm{f}}^{0+}: B_{1}^{0+} & =0, \\
B_{3}^{00} & =\frac{2}{\sqrt{6}} \frac{1}{P^{2}}\left[\nu^{2}-P^{2} Q^{2}\right] \sqrt{\left(Q^{2}+\frac{1}{4} P^{2}\right)^{2}-\nu^{2}} \\
B_{5}^{+0}: B_{1}^{+0} & =0, \\
B_{4}^{+0} & =-\frac{1}{\sqrt{2}} \nu \sqrt{P^{2}}\left[\frac{1}{2} \nu-Q^{2}\right] \sqrt{-Q^{2}-\nu-\frac{1}{4} P^{2}} \\
B_{4}^{00} & =-\frac{2}{\sqrt{6}} \nu^{2} \sqrt{\left(Q^{2}+\frac{1}{4} P^{2}\right)^{2}-\nu^{2}}, \\
& =\frac{1}{\sqrt{6}} P^{2} \sqrt{\left(Q^{2}+\frac{1}{4} P^{2}\right)^{2}-\nu^{2}} .
\end{aligned}
$$




$$
\begin{aligned}
& B_{4}^{0+}=\frac{1}{\sqrt{2}} \nu \sqrt{P^{2}}\left[\frac{1}{2} \nu+Q^{2}\right] \sqrt{-Q^{2}+\nu-\frac{1}{4} P^{2}} \\
& B_{5}^{0+}=-\frac{1}{\sqrt{2}} \sqrt{P^{2}}\left[\nu+\frac{1}{2} P^{2}\right] \sqrt{-Q^{2}+\nu-\frac{1}{4} P^{2}}
\end{aligned}
$$

We would like to acknowledge discussions with G. Preparata and a communication from R. Kingsley. One of us (P.Z.) expresses his gratitude to H. Joos, H. Schopper and G. Weber for the hospitality extended to him at DESY. G.K. would like to thank G. Kramer for a stay at the II. Institut für Theoretische Physik of the University of Hamburg while this work was being completed.

\section{References}

[1] H.W. Kendall, Deep Inelastic electron scattering in the continuum region, Proc. 1971 Int. Symp. on electron and photon interactions at high energies, Cornell University, Ithaca, N.Y. 1972.

[2] R. Brandt and G. Preparata, Nucl. Phys. B27 (1971) 541;

Y. Frishman, Phys. Rev. Letters 25 (1970) 266.

[3] H. Fritzsch and M. Gell-Mann, Light cone current algebra, Proc. Int. Conf. on duality and symmetry in hadron physics; Tel Aviv, 1971;

D.J. Gross and S.B. Treiman, Phys. Rev. D4 (1971) 1079.

[4] R.P. Feynman, Photon-hadron interactions (Benjamin, 1972);

S.D. Drell, D.J. Levy and T.M. Yan, Phys. Rev. D1 (1970) 1035, 1617, 2402;

P.V. Landshoff, J.C. Polkinghorne and R.D. Short, Nucl. Phys. B28 (1971) 210;

J.D. Bjorken and E.A. Paschos, Phys. Rev. 185 (1969) 1975.

[5] J.D. Bjorken Phys. Rev. 148 (1966) 1467;

N. Cabibbo, G. Parisi, M. Testa, Nuovo Cimento Letters 4 (1970) 35.

[6] T.F. Walsh and P. Zerwas, Nucl. Phys. B41 (1972) 551;

H. Terazawa, Phys. Rev. D5 (1972) 2259;

Z. Kunszt, Phys. Lett. 40B (1972) 220;

T.F. Walsh, Phys. Rev. D7 (1973) 2152;

H. Terazawa, Rev. Mod. Phys. 45 (1973) 615.

[7] S.J. Brodsky, Radiative problems and quantum electrodynamics, Proc. of the 1971 Symp. on electron and photon interactions at high energies, Cornell University, Ithaca, N.Y. 1972.

[8] C.E. Carlson and W.-K. Tung, Phys. Rev. D4 (1971) 2873;

R. Brown and I. Muzinich, Phys. Rev. D4, (1971) 1496.

[9] T.F. Walsh, see ref. [6].

[10] D.J. Gross and S.B. Treiman, Phys. Rev. D4 (1971) 2105

[11] R.L. Kingsley, Nucl. Phys. B36 (1972) 575;

A. Efremov and I.V. Ginzburg, Phys. Letters 36B (1971) 371.

[12] M. Bohm, H. Joos and M. Krammer, DESY preprint $72 / 62$;

G. Preparata, Phys. Rev. D7 (1973) 2973.

[13] R. Brandt and G. Preparata, Phys. Rev. Letters 25 (1970) 1530.

[14] R. Brandt and G. Preparata, see ref. [13];

G. Preparata, Erice Lectures, 1972. 
[15] P. Carruthers, Phys. Reports 1 (1970) 1.

[16] T.-M. Yan, Phys. Rev. D4 (1971) 3523.

[17] B. Renner, Nucl. Phys. B30 (1971) 634

[18] T.F. Walsh, Phys. Letters 36B (1971) 121;

S. Brodsky, T. Kinoshita and H. Terazawa, Phys. Rev. Letters 27 (1971) 280;

T.F. Walsh, and P. Zerwas, Phys. Letters 44B (1973) 195.

[19] B. Schrempp-Otto, F. Schrempp and T.F. Walsh, Phys. Letters 36B (1971) 463.

[20] K. Wilson, Currents, Proc. of the 1971 Int. Sympo on Electron and photon interactions at high energies, Cornell University, Ithaca, N.Y. 1972.

[21] H. Suura, T.F. Walsh and B.-L. Young, Nuovo Cimento Letters 4 (1972) 505.

[22] R.L. Kingsley, DAMTP preprint 73/14 (May, 1973). 